\title{
Flatly Broadened and Highly Coherent Supercontinuum Generation by Induced Modulation Instability in ANDi-PCF
}

\author{
Chunfu Cheng1,2, Yan Zeng1,2, Yiwen Ou ${ }^{1,2^{*}}$, Zhangyong Yang1,2, Zehao Chen ${ }^{1,2}$ \\ ${ }^{1}$ Hubei Collaborative Innovation Center for High-efficiency Utilization of Solar Energy, Hubei University of Technology, Wuhan, \\ China \\ ${ }^{2}$ School of Science, Hubei University of Technology, Wuhan, China \\ Email: *oyiwen@126.com
}

How to cite this paper: Cheng, C.F., Zeng, Y., Ou, Y.W., Yang, Z.Y. and Chen, Z.H. (2018) Flatly Broadened and Highly Coherent Supercontinuum Generation by Induced Modulation Instability in ANDi-PCF. Journal of Applied Mathematics and Physics, 6, 640-646.

https://doi.org/10.4236/jamp.2018.64056

Received: November 22, 2017

Accepted: April 9, 2018

Published: April 12, 2018

\begin{abstract}
In this paper, a flatly broadened and highly coherent supercontinuum generation by induced modulation instability in ANDi-PCF is proposed. The numerical results show that the bandwidth of supercontinuun can be increased by increasing the modulation depth and the coherence property of supercontinuum can be improved with the optimal modulation frequency. A highly coherent supercontinuum with the $10 \mathrm{~dB}$ bandwidth of $1305 \mathrm{~nm}$ and the flatness of less than $10 \mathrm{~dB}$ can be obtained when seeding at the modulation depth of 0.3 and modulation frequency of $24 \mathrm{THz}$.
\end{abstract}

\section{Keywords}

Supercontinuum Generation, Flat, Coherent, Modulation Instability

\section{Introduction}

Supercontinuum (SC) is a physical phenomenon in which a large intensity ultrashort optical pulse passes through a nonlinear medium, and the spectrum is greatly broadened due to the interaction of nonlinear effect and dispersion effect. SC spectrum is wide, relatively stable, simple and inexpensive, so it has been widely used in spectral analysis, optical coherence photography and optical sensing [1]. However, many applications of SC are limited by the coherence property of SC. For examples, If the coherence degree is not high, the resolution of the nonlinear microscope will be reduced; in the optical coherence tomography system (OCT), the coherence degree determines the measurement resolution level [2]; in terms of optical pulse compression, SC coherence degree di- 
rectly affects the quality of pulse compression [3] [4], and so on. Therefore, the coherence property of SC is very important which will ultimately affect the quality and range of the end application.

In contrast to the normal dispersion region, the anomalous dispersion region can produce a wider SC spectrum, but its coherence properties are very low and it is difficult to be used in many practical applications [1] [2] [3] [4]. In recent years, with the rapid development of the high nonlinear photonic crystal fiber (PCF), ultra-wide and highly coherent SC has become a possibility in the normal dispersion region. By using the numerical simulation and experimental method, the researchers have extensively studied the generation of coherent SC. During 2002-2003, J. M. Dudley [5] and X. Gu [6] analyzed the coherence prosperities of the SC by using the method of first-order factor. In 2010 Alexander M. Heidt studied the SC generation in all-normal dispersion photonic crystal fiber (ANDi-PCF) and analyzed the coherence properties of SC [7]. A SC with flatness of $1 \mathrm{~dB}$ and bandwidth of $1080 \mathrm{~nm}$ was achieved. From 2012 to 2017, many research teams have studied the SC and rogue wave generation by induced modulation instability in photonic crystal fiber [8] [9] [10] and they have pointed out the bandwidth can be increased by induced modulation instability. However, the effect of modulation instability on the coherence properties of SC has not been investigated. In this paper, we applied the seed depth and frequency in a pulsed light source to achieve highly coherent SC in ANDi-PCF. The results show that this method can improve the flatness and coherence of SC.

\section{Principle and Theory}

In this study, pulse propagation in the PCF is simulated using the Runge-Kutta algorithm to solve the generalized nonlinear Schrodinger equation [11]

$$
\frac{\partial A}{\partial z}=\sum_{m \geq 2} \frac{i^{m+1} \beta_{m}}{m !} \frac{\partial^{m} A}{\partial \tau^{m}}-\frac{\alpha}{2} A+i \gamma\left(1+\frac{i}{\omega_{0}} \frac{\partial}{\partial \tau}\right)\left[A(z, \tau) \int_{-\infty}^{+\infty} d \tau^{\prime} R\left(\tau-\tau^{\prime}\right)\left|A\left(z, \tau^{\prime}\right)\right|^{2}\right](1)
$$

where $A(z, \tau)$ is the complex field envelope at a propagation distance $z$ in a retarded reference time frame $\tau$ traveling at the envelope group velocity. Since only short pieces of the PCF will be considered, loss in the fiber is neglected $(\alpha=0)$. The dispersion coefficients $\beta_{m}$ are associated with the Taylor expansion of the propagation constant $\beta(w)$ around the center frequency $w_{0}$, and $\gamma$ is the frequency dependent nonlinear coefficient. Dispersion effects are described by the first term on the right hand side of Equation (1); nonlinear effects correspond to the second one. The nonlinear response function $R(t)=\left(1-f_{R}\right) \sigma(t)+f_{R} h_{R}(t)$ with $f_{R}=0.18$ contains both instantaneous and delayed Raman contributions, the response $h_{R}$ which we use is the same as the analytic approximation in [1].

As Dudley has pointed out the coherence property of supercontinuum can be improved by using shorter pulses [5]. Therefore, here we simulate the flatly broadened and highly coherent SC generation in the ANDi-PCF designed by P. S. Maji [12] by using 50-fs (full width at half maximum (FWHM) duration) linearly polarized pulse. At the wavelength of $1550 \mathrm{~nm}$, the nonlinear coefficient is 
estimated to be $\gamma=27.3\left(\mathrm{~W}^{-1} \cdot \mathrm{km}^{-1}\right)$, and the up to 8-th order dispersion coefficients are $\beta_{2}=6.9276 \times 10^{-2} \mathrm{ps}^{2} / \mathrm{km}, \quad \beta_{3}=-3.59804 \times 10^{-3} \quad \mathrm{ps}^{3} / \mathrm{km}$, $\beta_{4}=6.57756 \times 10^{-4} \mathrm{ps}^{4} / \mathrm{km}, \quad \beta_{5}=2.82896 \times 10^{-6} \mathrm{ps}^{5} / \mathrm{km}, \quad \beta_{6}=2.82896 \times 10^{-10}$ $\mathrm{ps}^{6} / \mathrm{km}, \quad \beta_{7}=1.45745 \times 10^{-12} \mathrm{ps}^{7} / \mathrm{km}, \quad \beta_{8}=2.91289 \times 10^{-14} \mathrm{ps}^{8} / \mathrm{km}$, the effect of more than 8 -th order dispersion can be neglected due to the weak dispersion effect of ANDi-PCF.

The modulated Gaussian input pulse envelope is assumed to have the following form

$$
A(z=0, \tau)=\left(\sqrt{P_{0}}+d_{0} \sqrt{P_{0}} e^{i 2 \pi f_{\bmod } \tau}\right) \exp \left(-\tau^{2} /\left(2 \tau_{p}^{2}\right)\right) \exp [i \delta \phi(\tau)]
$$

where $P_{0}=20 \mathrm{kw}$ is the input peak power and we define the power $P_{\text {seed }}=d_{0}^{2} P_{0}$ as the power of the seed, $d_{0}$ means the modulation depth, $f_{\bmod }$ is the modulation frequency, $\tau_{p}$ is the temporal width, which is related to FWHM pulse duration by $T_{F W H M} \approx 1.665 \tau_{p}, \delta \phi(\tau)$ is a small random phase fluctuation which is caused by Gaussian white noise [3] [4].

We take the Raman response function and high-order dispersion up to $\beta_{8}$ into account to obtain the MI gain $g(\Omega)$, it can be written as [8] [9] [10]:

$$
g(\Omega)=\operatorname{Im}\left\{\Delta k_{0} \pm \sqrt{\left(\Delta k_{e}+2 \gamma P_{0} \tilde{R}(\Omega)\right) \Delta k_{e}}\right\}
$$

where $\Omega$ is the modulation frequency, $\tilde{R}(\Omega)$ is Raman response for silica, $\Delta k_{e}$ and $\Delta k_{o}$ are sums over odd and even order derivatives of propagation constant $\beta$, respectively [8]:

$$
\Delta k_{0}=\sum_{m=1}^{\infty} \frac{\beta_{2 m+1}}{(2 m+1)} \Omega^{2 m+1}, \Delta k_{e}=\sum_{m=1}^{\infty} \frac{\beta_{2 m}}{2 m !} \Omega^{2 m},
$$

The coherence properties of SC spectra can be characterized by calculating the wavelength dependence of the first-order coherence as following equation [1].

$$
\left|g_{12}^{(1)}\left(\lambda, t_{1}-t_{2}\right)\right|=\left|\frac{<A_{1}^{*}\left(\lambda, t_{1}\right) A_{2}\left(\lambda, t_{2}\right)>}{\sqrt{<\left|A_{1}\left(\lambda, t_{1}\right)\right|^{2}><\left|A_{2}\left(\lambda, t_{2}\right)\right|^{2}>}}\right|,
$$

where the term in the angular brackets indicates an ensemble average over independently generated pairs of SC spectra through 20-times simulation results. The value of first-order coherence lies in the interval $[0,1]$, and a value of 1 denotes perfect coherence.

\section{Simulation and Discussion}

In order to obtain the coherent SC, we simulate the nonlinear propagation of an ultrashort pulse and SC generation in the ANDi-PCF with nearly zero flattened dispersion at $1550 \mathrm{~nm}$ by solving the nonlinear Schrödinger equation using the Runge-Kutta algorithm. Figure 1 shows the nonlinear propagation of an ultrashort laser pulse under the unseeded condition in ANDi-PCF at the input peak powers of $20 \mathrm{~kW}$. It can be seen that the pulse is not changed in the initial stage of pulse transmission as shown in Figure 1(b), which indicates that the effect of dispersion has no effect on the pulse propagation, the corresponding optical 


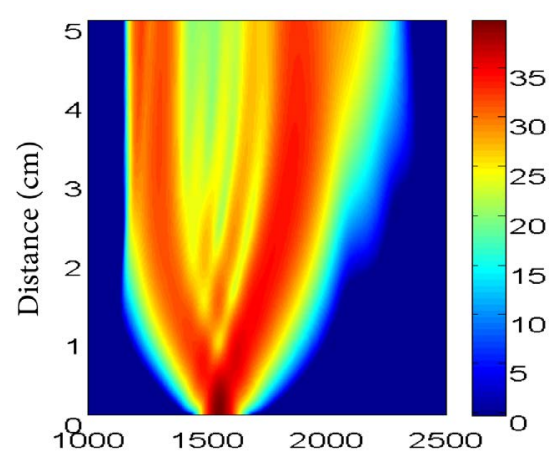

(a)

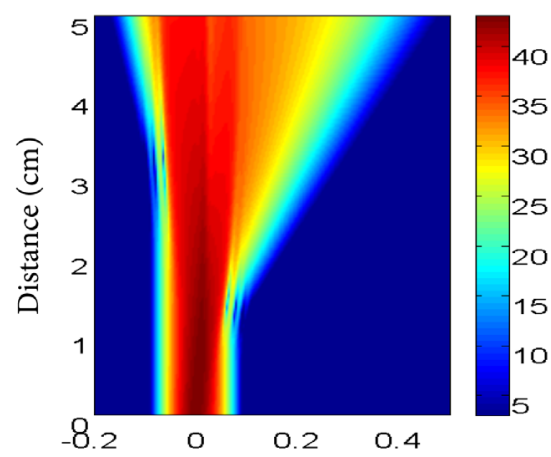

(b)

Figure 1. The propagation characteristics of an ultrashort laser pulse under the unseeded condition in ANDi-PCF. (a) spectral domain evolution; (b) time domain evolution. $\lambda_{0}=$ $1550 \mathrm{~nm}, \mathrm{P}_{0}=20 \mathrm{~kW}$.

spectra are symmetrically broadened due to SPM as shown in Figure 1(a). With increasing propagation distance, the splitting of the pulse and spectrum occurs due to the effect of dispersion and optical wave braking, which result in the asymmetry broadened of spectrum. Approximately at $4 \mathrm{~cm}$, the spectrum reaches almost its maximum bandwidth and a steady SC spectrum can be obtained after propagation distance of $4 \mathrm{~cm}$. At the propagation distance of $5 \mathrm{~cm}$, a highly coherent SC spectrum extending from about $1160 \mathrm{~nm}$ to $2311 \mathrm{~nm}$ at $10 \mathrm{~dB}$ level is generated as shown in Figure 2. In order to obtain a flatly broadened and highly coherent SC, the nonlinear propagation of an ultrashort laser pulse under the seeded condition in ANDi-PCF is also simulated. Figure 2 shows the effect of modulation depth on the SC spectrum and its coherence property under other conditions keep unchanged as in Figure 1. It can be seen that the bandwidth of spectrum can be further increased as the modulation depth increases. However, the flatness and the coherence property of SC spectrum became worse as the modulation depth increases; that is to say, the moderate modulation depth is a good choice to obtain wide-band SC with highly coherence. In order to improve the coherence property of SC, the effect of modulation frequency on the SC is simulated as shown in Figure 4 under the moderate modulation depth of 0.3 while the other parameters keep unchanged as in Figure 3. From Figure 4 we can see the most interesting effect of modulation frequency is that there exists an optimal modulation frequency to generate a flatly broadened and highly coherent SC. At the optimal modulation frequency of $24 \mathrm{THz}$, a highly coherent SC with the $10 \mathrm{~dB}$ bandwidth of $1305 \mathrm{~nm}$ and the flatness of less than $10 \mathrm{~dB}$ is obtained. In contrast, when the modulation frequency deviates the optimal modulation frequency, the flatness and the coherent property became worse, especially at the modulation frequency of $14 \mathrm{THz}$ the flatness and the coherent property is the worst. The reason that there exists an optimal modulation frequency can be explained as follows. When the modulation frequency is less than $14 \mathrm{THz}$, the generation mechanisms of SC are MI and SPM. In this case the collision between the seed and pump pulse leads to energy transferred to the parts 


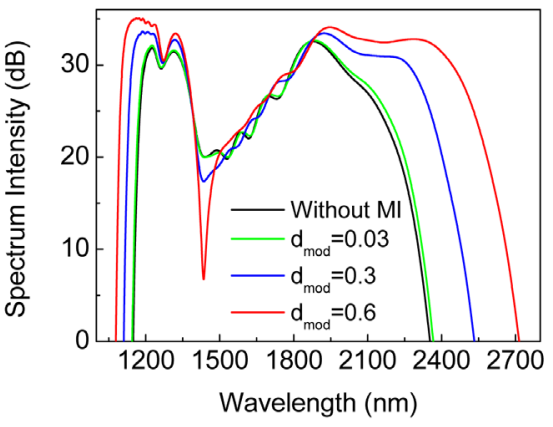

(a)

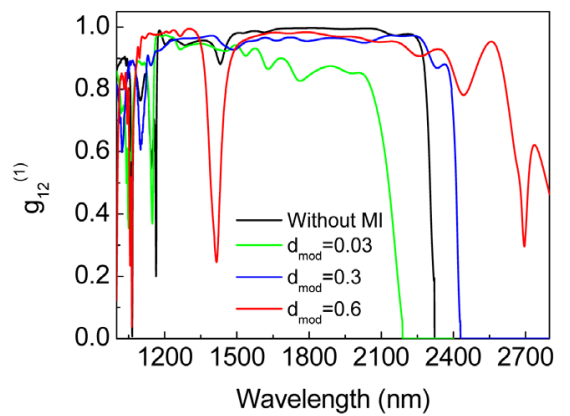

(b)

Figure 2. Effect of modulation depth on the (a) SC spectrum and (b) its coherence property.

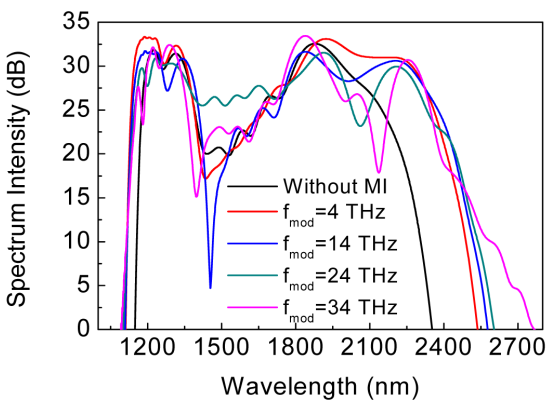

(a)

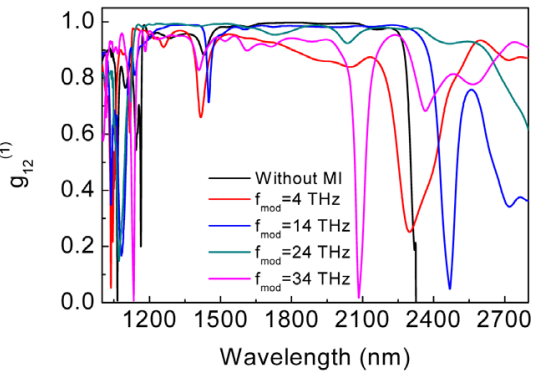

(b)

Figure 3. Effect of modulation frequency on the (a) SC spectrum and (b) its coherence property.

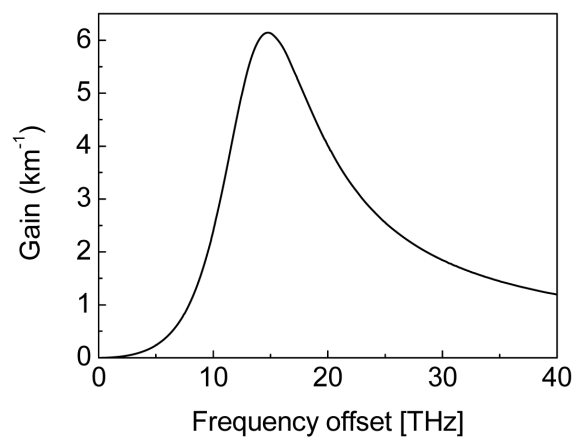

Figure 4. MI gain spectrum for the pump power of $20 \mathrm{~kW}$ as the function of seed frequency offset.

of short wavelength side, for example, when the modulation frequency is $14 \mathrm{THz}$ near the peak of gain spectrum as shown in Figure 4, more energy is transferred to the parts of short wavelength and thus forms a big dip as shown in Figure 3(a), which also degenerate the coherent property as shown in Figure 3(b). When the frequency is above the peak of gain spectrum, the generation mechanisms of SC are FWM and SPM [8], what's more, the FWM process is very weak and very weak FWM sidebands are generated on the both side of the pump as shown in Figure 5(a). In this case, the SC is generated mainly by SPM and the 


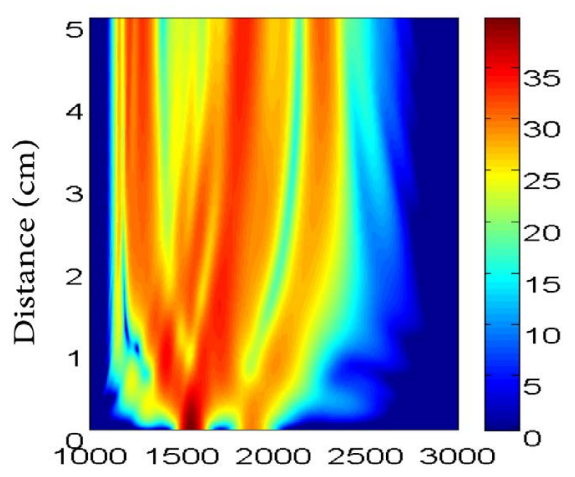

(a)

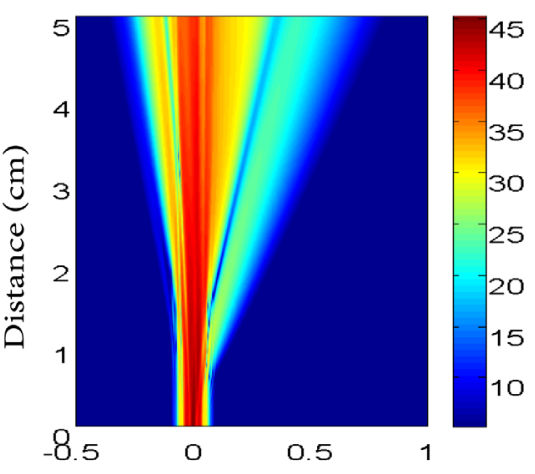

(b)

Figure 5. The propagation characteristics of an ultra short laser pulse under the seeded condition with a seed of 0.3 modulation depth at the modulation frequency of $24 \mathrm{THz}$. (a) spectral domain evolution; (b) time domain evolution.

formed the flat and highly coherent SC spectrum as shown in Figure 3. When the modulation frequency erty as shown in Figure 3(b). When the frequency is above the peak of gain spectrum, the generation mechanisms of SC are FWM and SPM [8], what's more, the FWM process is very weak and very weak FWM sidebands are generated on the both side of the pump as is too large, the work off effect occurs between the seed and pump pulse and thus leads to the flatness and coherence of SC spectrum became worse.

\section{Conclusion}

In conclusion, the nonlinear propagation and SC generation is numerically investigated. The results show that the flatness and coherence of SC can be improved by seeding with a proper modulation frequency and the bandwidth of SC can be increased by increasing the modulation depth. By selecting the optimal modulation parameters, a highly coherent supercontinuum with the $10 \mathrm{~dB}$ bandwidth of $1305 \mathrm{~nm}$ and the flatness of less than $10 \mathrm{~dB}$ can be obtained.

\section{Acknowledgements}

This work was supported by the National Nature Science Foundation of China (Grant Nos. 61475044), the International Science \& Technology Cooperation Program of China (No. 2016YFE0124300), the Doctoral Scientific Research Foundation of Hubei University of Technology (No. BSQD2016047) and the Open Foundation of Hubei Collaborative Innovation Center for High-efficiency Utilization of Solar Energy (No. HBSKFZD2017003).

\section{References}

[1] Dudley, J.M., Genty, G. and Coen, S. (2006) Supercontinuum Generation in Photonic Crystal Fiber. Rev. Mod. Phys., 78, 1135-1184.

https://doi.org/10.1103/RevModPhys.78.1135

[2] Hossaina, M.A., Namihiraa, Y., Razzakb, S.M.A., Islamc, M.A., Liud, J., Kaijagea, S.F. and Hirako, Y. (2012) Design of All Normal Dispersion Highly Nonlinear Pho- 
tonic Crystal Fibers for Supercontinuum Light Sources: Applications to Optical Coherence Tomography Systems. Opti. \& laser technol., 44, 976-980. https://doi.org/10.1016/j.optlastec.2011.10.021

[3] Foster, M.A., Gaeta, A.L., Cao, Q. and Trebino, R. (2005) Soliton-Effect Compression of Supercontinuum to Few-Cycle Durations in Photonic Nanowires. Opt. EXpress, 13, 6848-6855.https://doi.org/10.1364/OPEX.13.006848

[4] Cheng, C., Zeng, Y., Ou, Y., Lv, H., Lv, Q. and Zhang, J. (2017) Few-Cycle Pulse Generation from Compression of Supercontinuum by Optimizing Initial Chirp in ANDi-PCF. Optik, 133, 66-72. https://doi.org/10.1016/j.ijleo.2017.01.001

[5] Dudley, J.M. and Coen, S. (2002) Coherence Properties of Supercontinuum Spectra Generated in Photonic Crystal and Tapered Optical Fibers. Opt. Lett., 27, 1180-1182. https://doi.org/10.1364/OL.27.001180

[6] Gu, X., Kimmel, M., Shreenath, A.P., Trebino, R., Dudley, J.M., Coen, S. and Windeler, R.S. (2003) Experimental Studies of the Coherence of Microstructure-Fiber Supercontinuum. Opt. Express, 11, 2697-2703. https://doi.org/10.1364/OE.11.002697

[7] Heidt, A.M. (2010) Pulse Preserving Flat-Top Supercontinuum Generation in All-Normal Dispersion Photonic Crystal Fibers. J. Opt. Soc. Am. B, 27, 550-559. https://doi.org/10.1364/JOSAB.27.000550

[8] Sørensen, S.T., Larsen, C., Møller, U., Moselund, P.M., Thomsen, C.L. and Bang, O. (2012) Influence of Pump Power and Modulation Instability Gain Spectrum on Seeded Supercontinuum and Rogue Wave Generation. J. Opt. Soc. Am. B, 29, 2875-2885. https://doi.org/10.1364/JOSAB.29.002875

[9] Yang, H., Chen, N., Wang, B., Tang, P. and Zeng, Q. (2016) Supercontinuum and Rogue Soliton Generation by Induced Modulational Instability in Photonic Crystal Fibre. J. Mod. Optic., 63, 1370-1377. http://dx.doi.org/10.1080/09500340.2016.1148210

[10] Zhao, S., Yang, H., Chen, N. and Zhao, C. (2017) Controlled Generation of High-Intensity Optical Rogue Waves by Induced Modulation Instability. Scientific Reports, 7, 3926. https://doi:10.1038/srep39926

[11] Agrawall, G.P. (2001) Nonlinear Fiber Optics. Academic Press, New York.

[12] Maji, P.S. and Chaudhuri, P.R. (2014) A New Design for All-Normal Near Zero Dispersion Photonic Crystal Fiber with Selective Liquid Infiltration for Broadband Supercontinuum Generation at $1.55 \mu \mathrm{m}$. Journal of Photonics, 2014, 728592. http://dx.doi.org/10.1155/2014/728592 